\title{
Synthesis of 3-Aryl Coumarin Derivatives Using Ultrasound
}

\author{
Shubashini Krishnan Sripathi ${ }^{1,2^{*}}$, Kandasamy Logeeswari ${ }^{2}$ \\ ${ }^{1}$ Avinashilingam Institute for Home Science and Higher Education for Women, Tamilnadu, South India \\ ${ }^{2}$ Department of Chemistry, Avinashilingam Institute for Homescience and Higher Education for Women-University, \\ Coimbatore, India \\ Email: *adusks@gmail.com
}

Received January 2, 2013; revised February 10, 2013; accepted February 21, 2013

\begin{abstract}
Coumarins occupy an important place in the realm of natural products and synthetic organic chemistry. A fast and highly efficient green method for synthesizing 3-aryl coumarin derivatives from salicylaldehyde and phenyl acetyl chloride in the presence of tetrahydrofuran and $\mathrm{K}_{2} \mathrm{CO}_{3}$ using ultrasound irradiation is reported. Ultrasound assisted reactions have resulted in better yields and faster reaction time of the desired products than when prepared under conventional conditions. The resulting coumarin derivatives were characterized by IR spectrum.
\end{abstract}

Keywords: 3-Aryl Coumarin; Tetrahydrofuran; $\mathrm{K}_{2} \mathrm{CO}_{3}$; Salicylaldehyde; Phenyl Acetyl Chloride; Ultrasound

\section{Introduction}

In developing green [1] synthetic strategies, Indian scientists mainly concentrate on avoiding environmentally non-compatible reagents, solid-phase synthesis, modification of synthetic routes to decrease the number of steps and increase overall yield, usage of newer catalysts and simplification of classical procedures of reaction. Chemists play a key role in the realization of the conditions for sustainable development and green chemistry may be their winning strategy. Green chemistry also known as sustainable chemistry, is an umbrella concept that has grown substantially since it fully emerged a decade ago. One of the principle aims of green chemistry is to bring the benefit of modern chemical manufacture to developing countries without imposing the environmental burden that has plagued the industrialized world, green chemistry protects the environment, not by cleaning up, but by inventing new chemistry and new processes that do not pollute.

Among the various innovative alternative technologies for synthesizing compounds, currently ultrasound technology is of immense use. Ultrasound is the use of frequencies above human hearing creates mini-implosions of liquid bubbles that form is solution to create a unique environment for chemical reactions. Diverse types of reactions [2-10] like substitutions, addition, oxidationreduction, hydrolysis, alkylation, coupling reaction, enzymatic synthesis etc., are improved in terms of rates and

"Corresponding author. yield by sonication. In view of the enormous potentialities of ultrasound aided synthesis of organic compounds, the present work is aimed at synthesis of a biologically important class of compounds, namely coumarins with the aid of ultrasound.

Coumarins [11-19] and their derivatives are a wellknown class of heterocycles, that are very attractive due to their extended spectral range, high emission quantum yields, versatiliy in a large number of applications and mainly investigated due to their important biological activities [20-26] anti-coagulant, anti-HIV, anti hyper proliferative, cytotoxic properties as well chemopreventive activity against cancer and due to their role as anti-tubercular, anti-histamic, anti-microbial, anti-inflammatory rodenticides and photodynamic activity.

\section{Results and Discussion}

Nine different 3-aryl coumarins have been prepared by a one-pot method using ultrasound irradiation and the results are summarized in the Table 1.

The yields of 3-arylcoumarins range from 7\%-98\% with the maximum yield of $98 \%$ obtained for 6-nitro-3(4'-nitrophenyl) coumarin. The yield of 3-(2',4'-dinitrophenyl)coumarin is also high $(96 \%)$. Presence of nitro substituents either at C-6 or C-6 and C-4' together or $\mathrm{C}-2^{\prime}$ and $\mathrm{C}-4$ ' together in the $\mathrm{A} / \mathrm{C}$ rings of the coumarin skeleton favour coumarin formation. However substitution of a lone nitro group at C-4' position does not give a good yield of the 3-aryl coumarin as also in the case of the 6-bromo-4'-nitro substituted 3-arylcoumarin. The 
Table 1. Synthesis of 3-Aryl coumarin derivatives under ultrasound irradiation in the presence of THF and potassium carbonate.

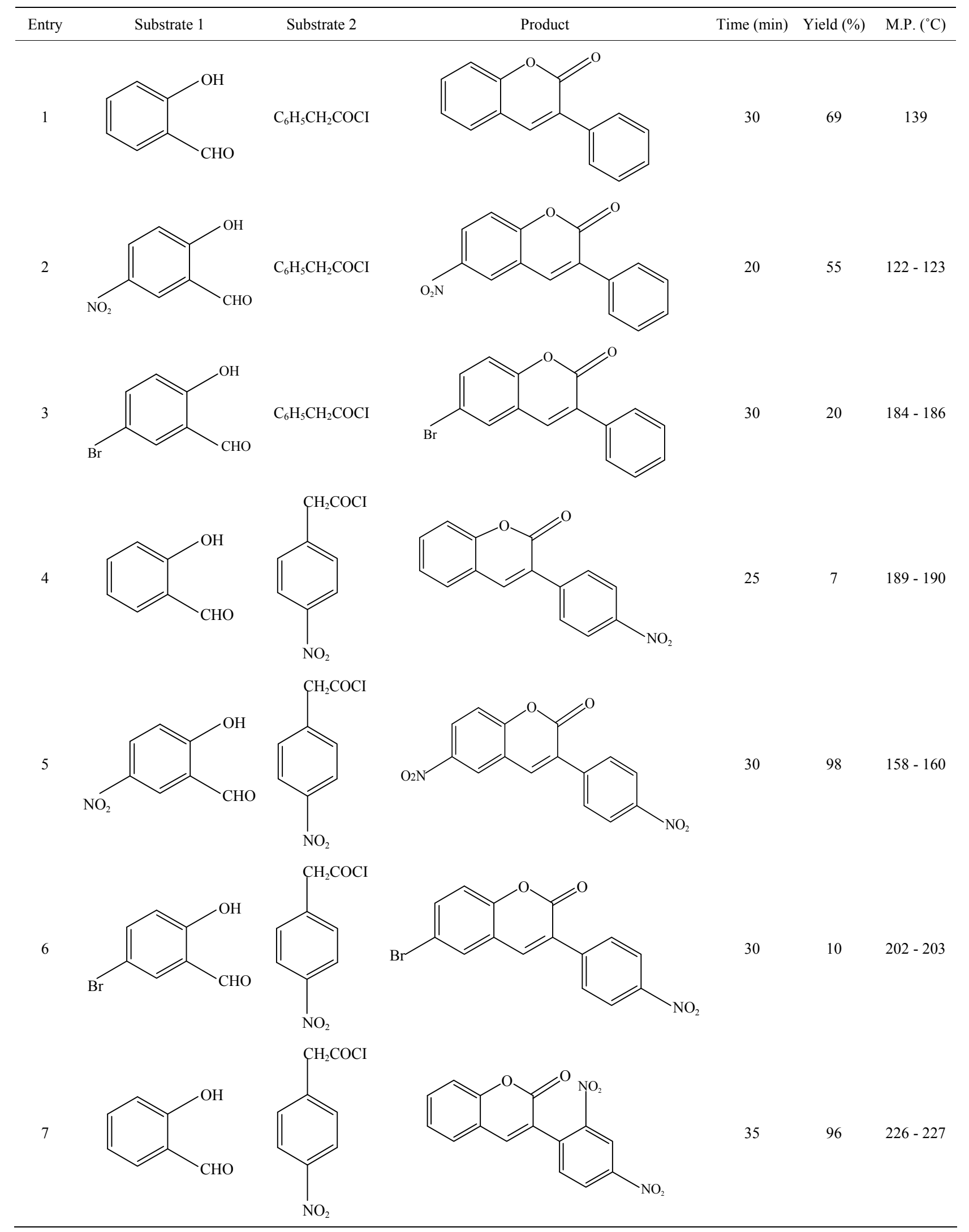


8<smiles>O=Cc1cc([N+](=O)[O-])ccc1O</smiles><smiles>O=Cc1cc(Br)ccc1O</smiles><smiles>O=C(Cl)Cc1ccc([N+](=O)[O-])cc1</smiles><smiles>O=c1oc2ccc([N+](=O)[O-])cc2cc1-c1ccc([N+](=O)[O-])cc1[N+](=O)[O-]</smiles>

$\mathrm{Br}$<smiles>O=c1oc2ccc(Br)cc2cc1-c1ccc([N+](=O)[O-])cc1[N+](=O)[O-]</smiles>

melting points were determined by open capillary method and are uncorrected.

The reaction time (Table 2) was found to be much minimized compared the conventional method of refluxing the reactants in dry acetone medium. The resulting compounds were recoded using IR spectrum.

1) 3-phenyl coumarin. The IR spectrum revealed prominent absorptions due to lactone carbonyl IR ( $\mathrm{KBr}$ ) $\gamma_{\max }=1708 \mathrm{~cm}^{-1}$ as well as C-O-C linkage IR $(\mathrm{KBr}) \gamma_{\max }$ $=1224 \mathrm{~cm}^{-1}$.

2) 6-nitro-3-phenyl coumarin. The IR spectrum revealed prominent absorptions due to lactone carbonyl IR $(\mathrm{KBr}) \gamma_{\max }=1680-1700 \mathrm{~cm}^{-1}$ as well as C-O-C linkage IR $(\mathrm{KBr}) \gamma_{\max }=1272 \mathrm{~cm}^{-1}$.

3) 6-bromo-3-phenyl coumarin. The IR spectrum revealed prominent absorptions due to lactone carbonyl IR (KBr) $\gamma_{\max }=1716 \mathrm{~cm}^{-1}$ as well as $\mathrm{C}-\mathrm{O}-\mathrm{C}$ linkage IR (KBr) $\gamma_{\max }=1236 \mathrm{~cm}^{-1}$.

4) 3-(4'-nitro-phenyl) coumarin. The IR spectrum revealed prominent absorptions due to lactone carbonyl IR $(\mathrm{KBr}) \gamma_{\max }=1797 \mathrm{~cm}^{-1}$ as well as C-O-C linkage IR (KBr) $\gamma_{\max }=1224 \mathrm{~cm}^{-1}$.

5) 6-nitro-3-(4'-nitrophenyl) coumarin. The IR spectrum revealed prominent absorptions due to lactone carbonyl IR (KBr) $\gamma_{\max }=1706 \mathrm{~cm}^{-1}$ as well as C-O-C linkage IR $(\mathrm{KBr}) \gamma_{\max }=1268 \mathrm{~cm}^{-1}$.

6) 6-bromo-3-(4'-nitrophenyl) coumarin. The IR spectrum revealed prominent absorptions due to lactone carbonyl IR $(\mathrm{KBr}) \gamma_{\max }=1680-1700 \mathrm{~cm}^{-1}$ as well as C-O-C linkage IR (KBr) $\gamma_{\max }=1348 \mathrm{~cm}^{-1}$.

7) 3-(2',4'dinitro-phenyl) coumarin. The IR spectrum revealed prominent absorptions due to lactone carbonyl IR (KBr) $\gamma_{\max }=1797 \mathrm{~cm}^{-1}$ as well as C-O-C linkage IR $(\mathrm{KBr}) \gamma_{\max }=1248 \mathrm{~cm}^{-1}$.

8) 6-nitro-(2',4'dinitro-phenyl) coumarin. The IR spectrum revealed prominent absorptions due to lactone carbonyl IR (KBr) $\gamma_{\max }=1670-1700 \mathrm{~cm}^{-1}$ as well as C-O-C linkage IR $(\mathrm{KBr}) \gamma_{\max }=1270 \mathrm{~cm}^{-1}$.

9) 6-bromo-3-(2',4'dinitro phenyl) coumarin. The IR spectrum revealed prominent absorptions due to lactone carbonyl IR (KBr) $\gamma_{\max }=1690-1700 \mathrm{~cm}^{-1}$ as well as C-O-C linkage IR (KBr) $\gamma_{\max }=1213 \mathrm{~cm}^{-1}$.

\section{Materials and Methods}

The most powerful and efficient sonication systems are the ultrasonic horns that allow insertion of an ultrasonic probe into the reaction vessel, in direct contact with reactants and solvent. The minimize energy losses resulting from deadening and the power supplied can be much higher. The reactor vessel was made by an experienced glassblower from pyrex borosilicate glass and was designed to be resistant to vibrations, including stress fractures. An ultrasonic homogenizer model 300 VT was used to carry out the reactions. Power in watts and the time in seconds can be set conveniently using this homogenizer. IR spectra were recorded using a PerkinElmer spectrometer.

\subsection{Preparation of Starting Compound}

\subsubsection{2,4-Dinitro Phenyl Acetic Acid}

Phenyl acetic acid $25 \mathrm{~g}(0.184 \mathrm{~mol})$ was taken in a 500 $\mathrm{ml} \mathrm{RB}$ flask and the flask was cooled in running water. $250 \mathrm{ml}$ of fuming nitric acid was added from a suitably supported dropping funnel rather slowly at first and then more rapidly. The addition required about $15 \mathrm{~min}$. A reflux condenser was attached to the flask and the flask and the mixture was heated under reflux for one hour and then carefully poured into $500 \mathrm{ml}$ of cold water. Then the crude product was filtered at the pump and washed it with a little cold water. The resulting acid was recrystallised from $20 \%$ alcohol. The yield of pure 2,4-dinitro- 
Table 2. Comparison of reaction time of 3-phenylcoumarin prepared in ultrasound with that of other process improvement methods and conventional method.

\begin{tabular}{ccccccc}
\hline \multirow{2}{*}{ S. No } & \multirow{2}{*}{ 3-phenyl coumarin } & \multicolumn{5}{c}{ Reaction time } \\
\cline { 3 - 7 } & 3-phenyl coumarin & Conventional & PTC & Solid support & Microwave & Ultrasound \\
\hline 1 & $30 \mathrm{hr}$ & $25 \mathrm{~min}$ & $10 \mathrm{~min}$ & $16 \mathrm{~min}$ & $30 \mathrm{~min}$ \\
2 & 3-(2', ','-dinitro phenyl) coumarin & $\mathrm{NR}$ & $\mathrm{NR}$ & $\mathrm{NR}$ & $5 \mathrm{~min}$ & $35 \mathrm{~min}$ \\
3 & 3-(4'-nitro phenyl) comarin & $\mathrm{NR}$ & $\mathrm{NR}$ & $\mathrm{NR}$ & $11 \mathrm{~min}$ & $25 \mathrm{~min}$ \\
4 & 6-nitro-3 phenyl coumarin & NR & NR & NR & $7 \mathrm{~min}$ & $20 \mathrm{~min}$ \\
\hline
\end{tabular}

NR: Not Reported by earlier workers using this procedure.

phenyl acetic acid is $27 \mathrm{~g}(64 \%)$, m.p.: $183^{\circ} \mathrm{C}$.

\subsubsection{Phenyl Acetyl Chloride}

A mixture of phenylacetic acid (10 g) and thionyl chloride $(7.6 \mathrm{ml})$ was refluxed on a water bath for an hour. After refluxing, the excess thionyl chloride was removed by distillation and the remaining liquid was distilled under reduced pressure, when phenyl acetyl chloride was obtained as a pale pink coloured liquid, yield $5 \mathrm{ml}$, b.p. $96^{\circ} \mathrm{C}-98^{\circ} \mathrm{C} / 12 \mathrm{~mm}$.

\subsection{3. p-Nitro Phenyl Acetyl Chloride}

A mixture of p-nitro phenyl acetic acid (10 g) and thionyl chloride $(7.6 \mathrm{ml})$ was refluxed on a water bath for an hour. After refluxing, the excess thionyl chloride was removed by distillation and the remaining liquid was distilled under reduced pressure, when phenyl acetyl chloride was obtained. Yield $4.7 \mathrm{ml}$, b.p. $\left(95^{\circ} \mathrm{C}-96^{\circ} \mathrm{C} /\right.$ $12 \mathrm{~mm})$.

\subsubsection{2,4-Dinitro Phenyl Acetyl Chloride}

A mixture of 2,4-dinitro phenyl acetic acid (10 g) and thionyl chloride $(7.6 \mathrm{ml})$ was refluxed on a water bath for an hour. After refluxing the excess thionyl chloride was removed by distilled and the remaining liquid was distilled under reduced pressure, when phenyl acetyl chloride was obtained. Yield $4.5 \mathrm{ml}$, b.p. $\left(93^{\circ} \mathrm{C}-94^{\circ} \mathrm{C} /\right.$ $12 \mathrm{~mm})$.

\subsubsection{5-Nitro Salicylaldehyde}

Salicylaldehyde $(1 \mathrm{ml})$ was taken in a RB flask to which a mixture of nitric acid and acetic acid in the ratio 1:3 was adde and stirred well for $5 \mathrm{~min}$. Then the mixture was poured into ice-cold water when yellow crystals of 5-nitrosalicylaldehyde separated, which were recrystallised from ethanol. Yield $4.4 \mathrm{~g}, \mathrm{~m} / \mathrm{p} / 126^{\circ} \mathrm{C}$.

\subsubsection{5-Bromo Salicylaldehyde}

Salicylaldehyde $(1 \mathrm{ml})$ was taken in a RB flask to which add bromine is added drop by drop through the separateing funnel with constant shaking stand the flask in cold water. When all the bromine has been added then reflux it for an hour and white solid was obtained, Yield 4 g.m.p, $116^{\circ} \mathrm{C}-117^{\circ} \mathrm{C}$.

\section{Experimental Section}

\section{General Procedure-Synthesis of 3-Phenyl Coumarin}

Salicylaldehyde $(0.3 \mathrm{ml})$ and phenyl acetyl chloride $(1 \mathrm{ml})$ were mixed in a glass vessel and potassium carbonate was added. The reaction mixture was irradiated in ultrasonic probe. TLC was run every 3 minutes to check the progress of the reaction. Once the reaction was complete as shown by the appearance of an additional fluorescent spot and disappearance of reactant, ice bath was added to the residue. The residue was then extracted with ether. The ether layer was washed with sodium carbonate solution and then with water. The ether layer was then evaporated and treated with ethanol when 3-phenylcoumarin was obtained as pale white crystals.

Using this procedure nine 3-phenylcoumarins derivatives were synthesized from salicylaldehyde, 5-nitrosalicylaldehyde or 5-bromosalicylaldehyde as one of the substrate and phenyl acetyl chloride, p-nitro phenyl acetyl chloride or 2,5-dinitro phenyl acetyl chloride as the other substrate.

\section{Conclusion}

Ultrasound irradiation in synthesis has been recognized to have a green value and various 3-aryl coumarins have been prepared from easily accessible starting materials in about 15 - 30 minutes of reaction time. The method appeared convenient and simple with respect to the convenient heating. When the reactions were carried out in ultrasound a tremendous reduction in time was observed. From the results of the study, it can be proposed that this method can be adopted as a green method of synthesis of coumarin derivatives. Further work is aimed at studying the structure activity of the synthesized coumarins.

\section{Acknowledgements}

The Authors thank the authorities of Avinashilingam 
Institute for Home Science and Higher Education for Women, Coimbatore, for providing all facilities necessary for the study.

\section{REFERENCES}

[1] A. J. Fry and D. Herr, "Reduction of $\alpha, \alpha$ '-Dibromo Ketones by Ultrasonically Dispersed Mercury in Protic Solvents," Tehrahedron Letters, Vol. 19, No. 20, 1979, pp. 1721-1724. doi:10.1016/0040-4039(78)80026-4

[2] J.-L. Luche, C. Petrier, J.-P. Lansard and A. E. Greene, "Ultrasound in Organic Synthesis: A Simplified Preparation of Diarylzinc Reagents and Their Conjugate Addition to Alpha-Enones," Journal of Organic Chemistry, Vol. 48, No. 21, 1983, pp. 3837-3839. doi:10.1021/jo00169a055

[3] J. Li, L. J. Li, T. S. Li and J. Z. Wang, "Ultrasound-Promoted Synthesis of 5-Substituted and 5,5-Disubstituted Hydantoins," Indian Journal of Chemistry, Vol. 37, No. 3, 1998, pp. 298-300.

[4] M. Kidwai, "Green Chemistry in India," Pure and Applied Chemistry, Vol. 73, No. 8, 2001, pp. 1261-1263. doi:10.1351/pac200173081261

[5] K. Wei, H.-T. Gao and W.-D. Z. Li, "Facile Synthesis of Oxabicyclic Alkenes by Ultra Sonication-Promoted Diels Alder Cycloaddition of Furano Dienes," The Journal of Organic Chemistry, Vol. 69, No. 17, 2004, pp. 5763-5765. doi:10.1021/jo049210a

[6] S. Moon, L. Duchin and J. V. Cooney, "Application of Ultrasound to Organic Reactions: Ultrasonic Catalysis on Hydrolysis of Carboxylic acid Esters," Tetrahedron Letters, Vol. 20, No. 41, 1979, pp. 3917-3920. doi:10.1016/S0040-4039(01)86464-9

[7] T. J. Mason, "Ultrasound in Synthetic Organic Chemistry," Chemical Society Reviews, Vol. 26, 1997, pp. 443451. doi:10.1039/cs9972600443

[8] W. Oppolzer and P. Schneider, "Practical Preparation and Metallo-Ene Reactions of (2-Alkenylallyl)-magnesium Chlorides: Comparative Study of Magnesium Activation," Tetrahedron Letters, Vol. 25, No. 31, 1984, pp. 3305-3308. doi:10.1016/S0040-4039(01)81370-8

[9] B. H. Han and P. Boudjouk, "Organic Sonochemistry. Ultrasound-Promoted Coupling of Organic Halides in the Presence of Lithium Wire," Tetrahedron Letters, Vol. 22, No. 29, 1981, pp. 2757-2758. doi:10.1016/S0040-4039(01)90544-1

[10] B. H. Han and P. Boudjouk, "Organic Sonochemistry Ultrasound-Promoted Reaction of Zinc with $\alpha, \alpha$ '-Dibromo-o-xylene. Evidence for Facile Generation of o-Xylene," The Journal of Organic Chemistry, Vol. 47, No. 4, 1982, pp. 751-752. doi:10.1021/jo00343a035

[11] D. I. Brahmbhatt, C. N. Patel, V. P. Pandya and M. A. Patel, "Synthesis of Some 3-Phenyl-4-methyl-6-(6-arylpyridin-2-yl) and 3-Phenyl-4-methyl-6-(4,6-diarylpyridin2-yl) Coumarins," The Journal of Organic Chemistry, Vol. 43, 2004, pp. 2228-2230.

[12] V. K. Ahluwalia and S. Mehta, "Removal of 4-Hydroxy Group in 4-Hydroxy-3-phenylcoumarins: Formation of 3-Phenylcoumarins," The Journal of Organic Chemistry,
Vol. 16, 1978, pp. 977-979.

[13] D. Bogdal, "Coumarins: Fast Synthesis by Knoevenagal Condensation under Microwave Irradiation," Journal of Chemical Research, Synopses, Vol. 8, 1998, pp. 468-469. doi:10.1039/a801724g

[14] V. Singh, J. Singh, K. P. Kaur and G. L. Kad, “Acceleration of the Pechmann Reaction by Microwave Irradiation: Application to the Preparation of Coumarins," Journal of Chemical Research, Vol. 2, 1997, pp. 58-59. doi:10.1039/a605672e

[15] G. Tocco, M. Begala, G. Delogu, G. Meli, C. Picciau and G. Podda, "Soluble Polymer-Supported Synthesis of 4-Methyl Coumarin on Modified Poly(ethylene glycol)s," Synlett, Vol. 8, 2005, pp. 1296-1300. doi:10.1055/s-2005-865231

[16] I. P. Beletskaya, O. G. Ganina, A. V. Tsvetkov, A. Yu. Fedoror and J.-P. Finet, "Synthesis of 4-Heteroaryl-substituted Coumarins by Suzuki Cross-Coupling Reactions," Synlett, Vol. 15, 2004, pp. 2797-2799.

[17] K. B. L. Mathur and K. N. Sawhney, "Studies on Some Structural Aspects of 4-Hydroxy Coumarin: Further Extension of Meerwein's Diazo Reaction and a Substitution Reaction Involving 2-Chloro-2-methylbut-yne," Indian Journal of Chemistry, Vol. 14, 1976, pp. 518-521.

[18] M. Kotani, K. Yamamoto, J. Oymada, Y. Fujiwara and T. Kitamura, "A Convenient Synthesis of Coumarins by Palladium(II)-Catalyzed Reaction of Phenols with Propiolic Acids," Synthesis, Vol. 9, 2004, pp. 1466-1470. doi:10.1055/s-2004-822360

[19] M. J. Matos, S. Vazquez-Rodriguez, F. Borges and E. Uriarte, "Alternative Methodologies for the Synthesis of Substituted 3-Arylcoumarins: Perkin Reactions and Palladium-Catalyzed Synthesis," 14th International Electronic Conference on Synthetic Organic Chemistry (ECSOC-14), 1-30 November 2010.

[20] L. A. Shastri, M. Ghate, and M. V. Kulkarni, "Dual Fluorescence and Biological Evaluation of Paracetamol Ethers from 4-Bromomethyl Coumarins," Indian Journal of Chemistry, Vol. 43, No. 11, 2004, pp. 2416-2422.

[21] D. Olmedo, R. Sancho, L. M. Bedoya, J. L. Lopez-Perez, E. del Olmo, E. Munoz, J. Alcami, M. P. Gupta and A. San Feliciano, "3-Phenylcoumarins as Inhibitors of HIV-1 Replication," Molecules, Vol. 17, No. 8, 2012, pp. 9245-9257. doi:10.3390/molecules 17089245

[22] J. Yang, G.-Y. Liu, F. Dai, X.-Y. Cao, Y.-F. Kang, L.-M. Hu, J.-J. Tang, X.-Z. Li, Y. Li, X.-L. Jin and B. Zhou, "Synthesis and Biological Evaluation of Hydroxylated 3-Phenylcoumarins as Antioxidants and Anti-Proliferative Agents," Bioorganic \& Medicinal Chemistry Letters, Vol. 21, No. 21, 2011, pp. 6420-6425. doi:10.1016/j.bmcl.2011.08.090

[23] K. V. Sashidhara, A. Kumar, M. Chatterjee, K. B. Rao, S. Singh, A. K. Verma and G. Palit, "Discovery and Synthesis of Novel 3-Phenylcoumarin Derivatives as Antidepressant Agents," Bioorganic \& Medicinal Chemistry Letters, Vol. 21, No. 7, 2011, pp. 1937-1941. doi:10.1016/j.bmcl.2011.02.040

[24] B. S. Joshi, N. Viswanathan, C. L. Kaul and R. S. Grewal, "Evaluation of Some Naturally Occurring \& Synthetic 
Coumarins for Hypotensive Activity," Indian Journal of Chemistry, Vol. 19, 1980, pp. 495-499.

[25] A. Arnoldi, G. Farina, R. Galli, L. Merlini and M. G. Parrino, "Analogs of Phytoalexins. Synthesis of Some 3-Phenylcoumarins and Their Fungicidal Activity," Journal of Agricultural and Food Chemistry, Vol. 34, No. 2, 1986, pp. 185-188. doi:10.1021/jf00068a007
[26] M. J. Matos, D. Vina, C. Picciau, F. Orallo, L. Santana and E .Uriarte, "Synthesis and Evaluation of 6-Methyl-3phenylcoumarins as Potent and Selective MAO-B Inhibitors," Bioorganic \& Medicinal Chemistry Letters, Vol. 19, No. 17, 2009, pp. 5053-5055.

doi:10.1016/j.bmcl.2009.07.039 